\title{
The Bang-Bang Funnel Controller
}

\author{
Daniel Liberzon and Stephan Trenn
}

\begin{abstract}
A bang-bang controller is proposed which is able to ensure reference signal tracking with prespecified time-varying error bounds (the funnel) for nonlinear systems with relative degree one or two. For the design of the controller only the knowledge of the relative degree is needed. The controller is guaranteed to work when certain feasibility assumptions are fulfilled, which are explicitly given in the main results. Linear systems with relative degree one or two are feasible if the system is minimum phase and the control values are large enough.
\end{abstract}

\section{INTRODUCTION}

Consider the single-input, single-output nonlinear system

$$
\begin{aligned}
& \dot{x}=F(x, u), \quad x(0)=x^{0} \in \mathbb{R}^{n}, \\
& y=H(x),
\end{aligned}
$$

where $F: \mathbb{R}^{n} \times \mathbb{R} \rightarrow \mathbb{R}^{n}, n \in \mathbb{N}$, is locally Lipschitz continuous and $H: \mathbb{R}^{n} \rightarrow \mathbb{R}$ is continuous. For a given reference signal $y_{\text {ref }}: \mathbb{R}_{\geq 0} \rightarrow \mathbb{R}$, we would like to achieve approximate reference signal tracking with a bang-bang feedback controller, i.e. $u(t) \in\left\{U_{-}, U_{+}\right\}$for all $t \geq 0$ and some $U_{-}<U_{+}$. Furthermore, we are aiming for guaranteed transient behavior of the error signal $e:=y-y_{\text {ref }}$ in the sense that the controller guarantees strict time-varying error bounds given by a socalled funnel

$$
\mathcal{F}:=\left\{(t, e) \in \mathbb{R}_{\geq 0} \times \mathbb{R} \mid \varphi_{-}(t) \leq e \leq \varphi_{+}(t)\right\},
$$

where $\varphi_{ \pm}: \mathbb{R}_{\geq 0} \rightarrow \mathbb{R}$ is the prespecified (time-varying) error bound with $\varphi_{-}(t)<0<\varphi_{+}(t)$ for all $t \geq 0$, see also Figure 1 .

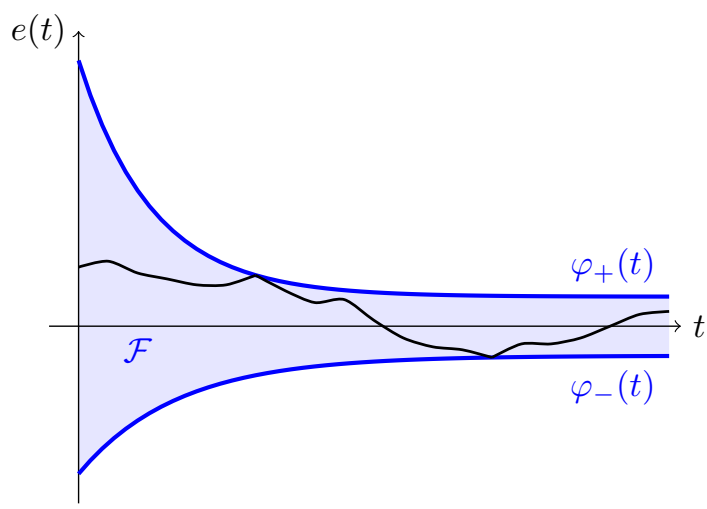

Fig. 1. The funnel $\mathcal{F}$ : A time-varying error bound.

The controller is governed by a switching logic whose output is a boolean variable $q: \mathbb{R}_{\geq 0} \rightarrow\{$ true, false $\}$ which

This work was supported by NFS grants CNS-0614993, ECCS-0821153 and DFG grant Wi1458/10-1

D. Liberzon is with the Coordinated Science Laboratory, University of Illinois at Urbana-Champaign, Urbana, IL 61801, USA and S. Trenn is with the Institute for Mathematics, University of Würzburg, 97074 Würzburg, Germany,

email: stephan.trenn@mathematik.uni-wuerzburg.de yields the control law

$$
u(t)= \begin{cases}U_{-}, & \text {if } q(t)=\text { true } \\ U_{+}, & \text {if } q(t)=\text { false. }\end{cases}
$$

The overall feedback system is illustrated in Figure 2.

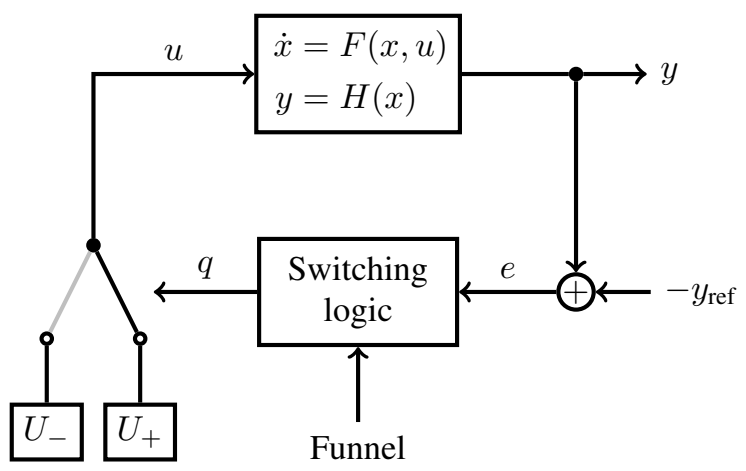

Fig. 2. Overall system structure.

The main purpose of this paper is twofold: On the one hand, we would like to find feasibility assumptions which take into account that, in general, an input signal with only two values cannot achieve arbitrary control objectives. On the other hand, we would like to find a switching logic which achieves the control objectives for feasible systems. We are aiming for a universal controller in the sense that the definition of the controller (given by the switching logic) does not involve the systems dynamics at all.

The feasibility assumption can be further distinguished into qualitative properties versus quantitative bounds of the systems dynamics. A qualitative property like the relative degree (see Definitions 2.1 and 3.1) yields different controller designs, while quantitative bounds do not influence the controller design but restrict the applicability of the controller. In this paper we will present controller designs for the relative degree one and two case.

We assume that the switching logic can also use derivatives of the error signal; in fact, for the relative degree two case the error $e$ and its derivative $\dot{e}$ is used to obtain the switching signal. For the relative degree one case only the error itself is needed.

Tracking control with prespecified strict time-varying error bounds has been studied first in [1] where the funnel controller was introduced. Funnel control itself is based on ideas from high-gain control and $\lambda$-tracking (see for example the survey [2]). There have been several extensions of the funnel controller, e.g. more general gain functions [3] and higher relative degree via backstepping and filters [4], [5]. A similar approach with a switched controller was proposed earlier in [6]; however, there the freedom to choose the time-varying bounds was more restricted and the gain was monotone. These results yielded universal controllers which were able to control all systems with some qualitative property 
(for example, relative degree one with stable zero dynamics). However, the price of the generality is that the input must be allowed to become arbitrarily large, which is problematic in applications. The first result concerning the funnel controller with input saturation was presented in [7] which was based on a model of an exothermic reactor. We will later use this model for illustrating the behavior of our bang-bang controller. Until now input saturation was only considered for the relative degree one case [8], [9]; input saturation for relative degree two systems is work in progress [10]. The design of the bang-bang funnel controller is inspired by the above results on funnel control with input saturation and the feasibility assumptions are very similar when $U_{ \pm}$are the bounds from the input saturation.

The key advantages of the bang-bang funnel controller in comparison to classical controllers are, firstly, the same advantages the continuous funnel controller from [1] has: no knowledge of the systems parameters is necessary for the controller design and prespecified transient behavior can be guaranteed. Secondly, in comparison to the continuous funnel controller, the bang-bang funnel controller is much simpler because it only uses two control values and doesn't need a time varying gain function. Furthermore, the bang-bang funnel controller is a piecewise linear system and certain aspects are therefore easier to analyze. Finally, the switching logic is still well defined when the error leaves the funnel; therefore, we believe the bang-bang funnel controller is also more robust to time delays (this is ongoing research). However, in applications where a continuous controller signal is desired or where the input should not be saturated all the time, the bang-bang funnel controller is not applicable.

Using the bang-bang controller governed by a discrete switching logic together with a continuous system yields a hybrid system (see e.g. [11] and the references therein for an overview). The prespecified error bounds given by the funnel can be seen as a time-varying generalization of the invariance problem for hybrid systems as studied e.g. in [12]. In general, the coupling of continuous and discrete dynamics could lead to problems concerning the existence of solutions; however, by implementing the switching logic with some hysteresis effect this solvability problem can be avoided. For switched systems (average) dwell times are important, because in practical applications arbitrarily fast switching is often not possible and from a mathematical point of view dwell times also exclude so-called Zeno-behavior (i.e. infinitely many switches in finite time). The main results in this paper also give conditions when the switching times of the control input have an (average) dwell time.

The structure of the paper is as follows. The main results for the relative degree one case are given in Sections II and the relative degree two case is considered in Section III. In both cases, first the precise meaning of relative degree is defined. Afterwards, the switching logic is given and some general properties of the closed loop are highlighted. Afterwards, the two main results, Theorem 2.4 and Theorem 3.4, are given. For the relative degree one case we apply the bang-bang funnel controller to an exothermic reactor model.

Throughout the paper we use $\|f\|$ for the supremum norm of the function $f: \mathbb{R} \rightarrow \mathbb{R}$; by $|x|$ for $x \in \mathbb{R}^{n}$ we denote the Euclidean vector norm and $|A|$ denotes the induced norm of the matrix $A \in \mathbb{R}^{m \times n}$. For defining predicates (i.e. for functions with values in the set $\{$ true, false $\}$ ) we use the notation [statement] $\in\{$ true, false $\}$. Solutions of differential equations are considered in the sense of Carathéodory, i.e. solutions are assumed to be absolutely continuous and fulfill the differential equation almost everywhere.

Due to space limitations we omit the proofs of all results and refer to the longer version of this paper [13].

\section{RELATIVE DEGREE ONE CASE}

Definition 2.1 (Relative degree one): The system (1) is said to have (global) relative degree one (with positive gain) when there exist locally Lipschitz continuous functions $f$ : $\mathbb{R} \times \mathbb{R}^{n-1} \rightarrow \mathbb{R}, h: \mathbb{R} \times \mathbb{R}^{n-1} \rightarrow \mathbb{R}^{n-1}$, continuous $g$ : $\mathbb{R} \times \mathbb{R}^{n-1} \rightarrow \mathbb{R}_{>0}$ and a diffeomorphism $\Phi: \mathbb{R}^{n} \rightarrow \mathbb{R} \times \mathbb{R}^{n-1}$, $x \mapsto(y, z)$ which equivalently transforms (1) to

$$
\begin{array}{ll}
\dot{y}=f(y, z)+g(y, z) u, & y(0)=y^{0}, \\
\dot{z}=h(y, z), & z(0)=z^{0},
\end{array}
$$

where $\left(y^{0}, z^{0}\right)=\Phi\left(x^{0}\right)$.

For the relative degree one case we propose the following simple switching logic:

$$
\begin{aligned}
q(0-) & =[e(0) \geq 0], \\
q(t) & =\mathfrak{S}\left(e(t), \varphi_{+}(t), \varphi_{-}(t), q(t-)\right),
\end{aligned}
$$

where $\mathfrak{S}: \mathbb{R} \times \mathbb{R} \times \mathbb{R} \times\{$ true, false $\} \rightarrow\{$ true, false $\}$ is the switching predicate given by

$$
\mathfrak{S}\left(e, \bar{e}, \underline{e}, q_{\mathrm{old}}\right):=\left[e \geq \bar{e} \vee\left(e>\underline{e} \wedge q_{\mathrm{old}}\right)\right] \text {. }
$$

Since $\varphi_{+}(t)>\varphi_{-}(t)$ for all $t \geq 0$ the switching logic (5) together with (3) can also be described by a state diagram as shown in Figure 3.

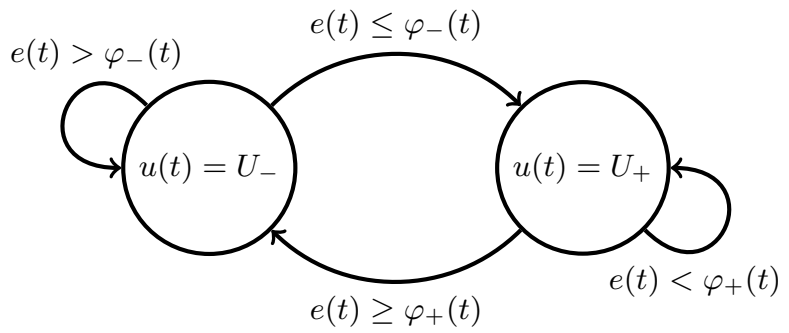

Fig. 3. The switching logic for the relative degree one case.

Note that for $\bar{e}>\underline{e}$, the following equivalence holds

$$
\mathfrak{S}\left(e, \bar{e}, \underline{e}, q_{\mathrm{old}}\right) \Leftrightarrow \neg \mathfrak{S}\left(-e,-\underline{e},-\bar{e}, \neg q_{\mathrm{old}}\right),
$$

which explains the symmetry in Figure 3.

The following lemma is essential to prove existence of solutions of the closed loop.

Lemma 2.2 (Well-defined causal switching logic): For every continuous error function $e:[0, T) \rightarrow \mathbb{R}, 0<T \leq \infty$, there exists a unique causal right-continuous switching signal $q:[0, T) \rightarrow\{$ true, false $\}$ fulfilling (5). Furthermore, if $e$ is absolutely continuous with right-continuous bounded derivative $\dot{e}$ and

$$
\inf _{t \geq 0} \varphi_{+}(t)+\inf _{t \geq 0}-\varphi_{-}(t):=\lambda_{+}+\lambda_{-}>0
$$

then the switching signal has a positive dwell time $\tau_{d}>0$, i.e. two switches of $q$ are at least $\tau_{d}$ apart. In fact

$$
\tau_{d} \geq \frac{\lambda_{+}+\lambda_{-}}{\|\dot{e}\|} .
$$


Note that in Lemma 2.2 we did not assume that the error evolves within the funnel. A direct consequence of Lemma 2.2 is the following result for the closed loop.

Corollary 2.3 (Closed loop well posed): Consider the system (1) in closed loop with the bang-bang controller given by (3) and (5), where $e:=y-y_{\text {ref }}$ for some continuous reference signal $y_{\text {ref }}: \mathbb{R}_{>0} \rightarrow \mathbb{R}$. Then for every initial value $x^{0} \in \mathbb{R}^{n}$ there exists a unique maximal solution $(x, q):[0, \omega) \rightarrow \mathbb{R}^{n} \times\{$ true, false $\}, 0<\omega \leq \infty$ of the closed loop.

Note that Corollary 2.3 neither claims nor assumes that the error signal evolves within the funnel, even finite escape time is not excluded at this point. To prove that the error evolves within the funnel, we need some additional feasibility assumptions which are formulated in the following theorem.

Theorem 2.4 (Relative degree one main result): Assume that (1) has relative degree one, i.e. (1) is equivalent to (4). Consider a funnel $\mathcal{F}$ as given by (2) and assume additionally that the funnel boundaries $\varphi_{ \pm}: \mathbb{R}_{\geq 0} \rightarrow \mathbb{R}$ as well as the reference signal $y_{\text {ref }}: \mathbb{R}_{\geq 0} \rightarrow \mathbb{R}$ are absolutely continuous with right-continuous derivatives. Assume that the initial conditions for (4) fulfill

$$
y^{0}-y_{\text {ref }}(0) \in\left[\varphi_{-}(0), \varphi_{+}(0)\right], \quad z^{0} \in Z_{0} \subseteq \mathbb{R}^{n-1}
$$

and assume that for every continuous $y:[0, \infty) \rightarrow \mathbb{R}$ with $\varphi_{-}(t) \leq y(t)-y_{\text {ref }}(t) \leq \varphi_{+}(t)$ for all $t \geq 0$ and all initial values $z^{0} \in Z_{0}$ there exist a unique (global) solution $z: \mathbb{R}_{\geq 0} \rightarrow \mathbb{R}^{n-1}$ of the zero dynamics (4b); for $t>0$ let

$Z_{t}:=\left\{\begin{array}{l|l}z(t) & \begin{array}{l}z:[0, t] \rightarrow \mathbb{R}^{n-1} \text { solves }(4 \mathrm{~b}) \text { for some } \\ z^{0} \in Z_{0} \text { and for some } y:[0, t] \rightarrow \mathbb{R} \text { with } \\ \varphi_{-}(\tau) \leq y(\tau)-y_{\mathrm{ref}}(\tau) \leq \varphi_{+}(\tau) \forall \tau \in[0, t]\end{array}\end{array}\right\}$.

If the feasibility conditions

$$
\begin{aligned}
& U_{-}<\frac{\dot{\varphi}_{+}(t)+\dot{y}_{\mathrm{ref}}(t)-f\left(y_{\mathrm{ref}}(t)+\varphi_{+}(t), z_{t}\right)}{g\left(y_{\mathrm{ref}}(t)+\varphi_{+}(t), z_{t}\right)} \\
& U_{+}>\frac{\dot{\varphi}_{-}(t)+\dot{y}_{\mathrm{ref}}(t)-f\left(y_{\mathrm{ref}}(t)+\varphi_{-}(t), z_{t}\right)}{g\left(y_{\mathrm{ref}}(t)+\varphi_{-}(t), z_{t}\right)}
\end{aligned}
$$

hold for all $t \geq 0$ and all $z_{t} \in Z_{t}$ then the closed loop composed of the system (1) or, equivalently, (4) and the bangbang controller (3) governed by the switching logic (5) has the following properties:

1) There exists a unique (global) solution $(x, q): \mathbb{R}_{\geq 0} \rightarrow$ $\mathbb{R}^{n} \times\{$ true, false $\}$.

2) The error $e:=y-y_{\text {ref }}$ evolves within the funnel, i.e. $(t, e(t)) \in \mathcal{F}$ for all $t \geq 0$.

$3)$ If $f$ and $g$ are uniformly bounded on $\bigcup_{t>0}\left[y_{\mathrm{ref}}(t)+\right.$ $\left.\varphi_{-}(t), y_{\text {ref }}(t)+\varphi_{+}(t)\right] \times Z_{t}, \dot{y}_{\text {ref }}$ is bounded and (7) holds then the jumping times of $u$ or, equivalently, the switches of $q$ have a positive dwell time $\tau_{d}>0$.

Remarks 2.5: 1) The feasibility conditions (8) can be simplified by using upper bounds for the funnel boundaries (and their derivatives), the zero dynamics, and the reference signal (and its derivative):

$$
U_{-}<-\frac{\left\|\dot{\varphi}_{+}\right\|+\left\|\dot{y}_{\mathrm{ref}}\right\|+F_{\max }}{G_{\min }}, U_{+}>\frac{\left\|\dot{\varphi}_{-}\right\|+\left\|\dot{y}_{\mathrm{ref}}\right\|+F_{\max }}{G_{\min }},
$$

where $\quad F_{\max }:=\max _{|y| \leq Y_{\max },|z| \leq Z_{\max }}|f(y, z)|$,

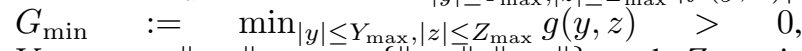
$Y_{\max }:=\left\|y_{\text {ref }}\right\|+\max \left\{\left\|\varphi_{+}\right\|,\left\|\varphi_{-}\right\|\right\}$and $Z_{\max }$ is an upper bound for the zero dynamics, i.e. all solutions of (4b) with $|y(t)| \leq Y_{\max }$, for all $t \geq 0$, fulfill $z(t) \leq Z_{\max }$ for all $t \geq 0$ (in particular, the initial value $z_{0}$ must be bounded by $Z_{\max }$ ). A consequence of considering this more conservative feasibility assumption is that $U_{-}<0$ and $U_{+}>0$ has to hold which is often too restrictive especially for nonlinear systems, see Example 2.6.

2) Consider a linear system with relative degree one in normal form [14] (see also [5, Lem. 3.5])

$$
\begin{array}{ll}
\dot{y}=\alpha y+s^{\top} z+\gamma u & y(0)=y^{0}, \\
\dot{z}=p y+Q z & z(0)=z^{0},
\end{array}
$$

where $\alpha \in \mathbb{R}, s, p \in \mathbb{R}^{n-1}, Q \in \mathbb{R}^{(n-1) \times(n-1)}$ and $\gamma>$ 0 . Assume that the initial value for the zero dynamics is bounded say by $M>0$. If the linear system is minimum phase, i.e. $Q$ is Hurwitz with $\left|e^{Q t}\right| \leq C e^{-\lambda t}, C, \lambda>0$, then boundedness of $y$ implies

$$
\begin{aligned}
|z(t)| & \leq C e^{-\lambda t}\left|z_{0}\right|+\int_{0}^{t} C e^{-\lambda(t-s)}|p||y(s)| \mathrm{d} s \\
& \leq C M+\frac{C}{\lambda} Y_{\max }=: Z_{\max } .
\end{aligned}
$$

Hence with $F_{\max }=|\alpha| Y_{\max }+\left|s^{\top}\right| Z_{\max }$ and $G_{\min }=\gamma$ the condition (9) is always fulfilled when $U_{-}<0$ and $U_{+}>0$ are large enough.

3) The sets $Z_{t} \subseteq \mathbb{R}^{n-1}, t \geq 0$, are defined by considering $y: \mathbb{R}_{\geq 0} \rightarrow \mathbb{R}$ as an input to the system governed by (4b). For the definition of $Z_{t}$ it is not assumed that $y$ solves the closed loop, it is merely assumed that $y$ evolved within the funnel on the interval $[0, t]$. For the feasibility assumptions (8), it is not needed that the sets $Z_{t}$ are uniformly bounded as long as $f$ and $1 / g$ do not get unbounded for unbounded $t \mapsto z_{t} \in Z_{t}$. In particular, it is therefore possible to apply the result also to timevarying systems by the common trick of including time as an additional differential equation $\dot{t}=1$.

4) The bang-bang controller works also when the funnel boundaries are not bounded away from zero; however, then the length of the switching intervals will converge to zero. The corresponding behavior for the continuous funnel controller from [1] is that the gain $k(t)$ grows unbounded (however, all continuous funnel controller results are only formulated for the case that the funnel boundaries are bounded away from zero). In contrast to the continuous funnel controller, this undesired behavior can already be excluded by assuming (7) which allows that one of the two funnel boundaries approaches zero. In fact, (7) can be further weakened (cf. (15)) such that both funnel boundaries can approach zeros, as long as they don't do it simultaneously.

Example 2.6: We consider a model of exothermic chemical reactions which was used in [7] to study the funnel control with input saturation. In the notation of the present paper the model with one reactant and one product reads as

$$
\begin{aligned}
\dot{y} & =b r\left(z_{1}, z_{2}, y\right)-q y+u, & y(0) & =y^{0}>0, \\
\dot{z}_{1} & =c_{1} r\left(z_{1}, z_{2}, y\right)+d\left(z_{1}^{\text {in }}-z_{1}\right), & z_{1}(0) & =z_{1}^{0} \geq 0, \\
\dot{z}_{2} & =c_{2} r\left(z_{1}, z_{2}, y\right)+d\left(z_{2}^{\text {in }}-z_{2}\right), & z_{2}(0) & =z_{2}^{0} \geq 0,
\end{aligned}
$$

where $b \geq 0, q>0, c_{1}<0, c_{2} \in \mathbb{R}, d>0, z_{1 / 2}^{\text {in }} \geq 0$ and $r$ : $\mathbb{R}_{\geq 0} \times \mathbb{R}_{\geq 0} \times \mathbb{R}_{>0} \rightarrow \mathbb{R}_{\geq 0}$ is assumed to be locally Lipschitz continuous with $r(0, T)=0$ for all $T>0$. The reference 
signal is $y_{\text {ref }}(t)=y^{*}>0$ for all $t \geq 0$. In [7] the input is saturated to some interval $\left[U_{-}, U_{+}\right]$with $U_{-}<U_{+}$, i.e. $u(t) \in\left[U_{-}, U_{+}\right]$for all $t \geq 0$, and the feasibility assumption in [7] is that there exists $\gamma \in \mathbb{R}_{>0}^{2}$ with $\left(c_{1}, c_{2}\right) \gamma \leq 0$ and

$$
\begin{aligned}
& \exists \rho_{-}, \rho_{+}>0 \exists \bar{y}>y^{*} \forall y \in\left[y^{*}, \bar{y}\right] \forall z_{1}, z_{2} \in Z_{0}: \\
& 0<U_{-}+\rho_{-}<q y-\operatorname{br}\left(z_{1}, z_{2}, y\right)<U_{+}-\rho_{+} .
\end{aligned}
$$

where $Z_{0}:=\left\{\left(z_{1}, z_{2}\right) \in \mathbb{R}_{>0}^{2} \mid\left(z_{1}, z_{2}\right) \gamma<\left(z_{1}^{\text {in }}, z_{2}^{\text {in }}\right) \gamma\right\}$. It can be shown that $Z_{0}$ is positively invariant for every $y: \mathbb{R} \rightarrow$ $\mathbb{R}_{>0}$. Hence, in the notation of Theorem 2.4, $Z_{t} \subseteq Z_{0}$ for all $t \geq 0$ if $\left(z_{1}^{0}, z_{2}^{0}\right) \in Z_{0}$. Now [7, Rem. 2] shows that for every funnel $\mathcal{F}$ whose funnel boundaries $\varphi_{ \pm}$fulfill

$$
\begin{array}{ll}
\varphi_{+}(t) \in\left(0, \bar{y}-y^{*}\right], & \varphi_{-}(t) \in\left(-y^{*}, 0\right), \\
\dot{\varphi}_{+}(t)>-\rho_{-}, & \dot{\varphi}_{-}(t)<\rho_{+},
\end{array}
$$

the feasibility assumption (8) holds. A simulation of the bangbang controller applied to the above model with parameters as given in [7, Sec. 3.3] is shown in Figure 4.
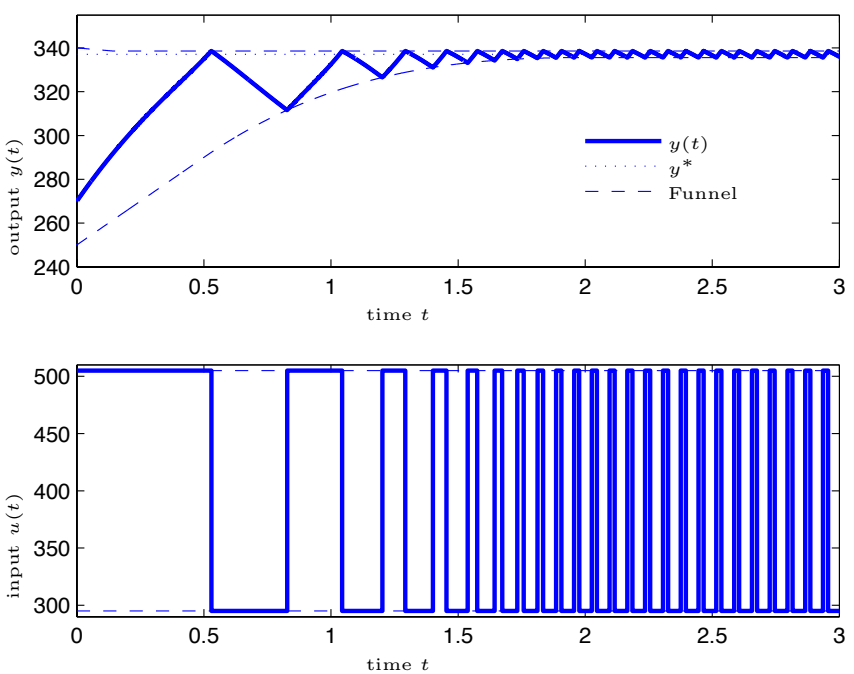

Fig. 4. The bang-bang funnel controller applied to an exothermic reactor model.

\section{RELATIVE DEGREE TWO CASE}

Definition 3.1 (Relative degree two): The system (1) is said to have (global) relative degree two (with positive gain) when there exist locally Lipschitz continuous functions $f$ : $\mathbb{R} \times \mathbb{R} \times \mathbb{R}^{n-2} \rightarrow \mathbb{R}, h: \mathbb{R} \times \mathbb{R} \times \mathbb{R}^{n-2} \rightarrow \mathbb{R}^{n-2}$, continuous $g: \mathbb{R} \times \mathbb{R} \times \mathbb{R}^{n-2} \rightarrow \mathbb{R}_{>0}$ and a diffeomorphism $\Phi: \mathbb{R}^{n} \rightarrow \mathbb{R} \times \mathbb{R} \times \mathbb{R}^{n-2}, x \mapsto(y, \dot{y}, z)$ which equivalently transforms (1) to

$$
\begin{array}{ll}
\ddot{y}=f(y, \dot{y}, z)+g(y, \dot{y}, z) u & y(0)=y^{0}, \dot{y}(0)=\dot{y}^{0}, \\
\dot{z}=h(y, \dot{y}, z) & z(0)=z^{0},
\end{array}
$$

where $\left(y^{0}, \dot{y}^{0}, z^{0}\right)=\Phi\left(x^{0}\right)$.

The switching logic for the relative degree two case requires a second funnel for $\dot{e}$ given by

$$
\mathcal{F}^{d}:=\left\{(t, \dot{e}) \in \mathbb{R}_{\geq 0} \times \mathbb{R} \mid \varphi_{-}^{d}(t) \leq \dot{e} \leq \varphi_{+}^{d}(t)\right\},
$$

where $\varphi_{-}^{d}(t)<0<\varphi_{+}^{d}(t)$ for all $t \geq 0$. The idea to use a derivative funnel originates from the recent work [10]. This funnel might reflect real physical bounds for $\dot{e}$ or might be used as a design parameter for the controller. Anyway, the derivative funnel $\mathcal{F}_{d}$ cannot restrict $\dot{e}$ in such a way that $e$ cannot decrease or increase fast enough to follow the boundaries of the original funnel $\mathcal{F}$; in fact it must hold that

$$
\forall t \geq 0: \quad \varphi_{+}^{d}(t)>\dot{\varphi}_{-}(t) \quad \text { and } \quad \varphi_{-}^{d}(t)<\dot{\varphi}_{+}(t),
$$

where we assumed that the funnel boundary functions $\varphi_{ \pm}$: $\mathbb{R}_{\geq 0} \rightarrow \mathbb{R}$ are absolutely continuous with right-continuous derivatives. In addition to the derivative funnel a "safety distance" $\varepsilon_{ \pm}>0$ from the corresponding funnel boundary $\varphi_{ \pm}$is needed to prevent the error $e$ from leaving the funnel $\mathcal{F}$. This distance will play an essential role in the feasibility assumptions later; at this point we already make the following assumption:

$$
\forall t \geq 0: \quad \varphi_{+}(t)-\varepsilon_{+}>0 \quad \text { and } \quad \varphi_{-}(t)+\varepsilon_{-}<0 .
$$

The switching logic is now given by $q_{0}(0-)=[e(0) \geq 0]$, $q(0-)=q_{0}(0-)$ and

$$
\begin{aligned}
q_{0}(t) & =\mathfrak{S}\left(e(t), \varphi_{+}(t)-\varepsilon_{+}, \varphi_{-}(t)+\varepsilon_{-}, q_{0}(t-)\right) \\
q(t) & = \begin{cases}\mathfrak{S}\left(\dot{e}(t), \min \left\{\dot{\varphi}_{+}(t), 0\right\}, \varphi_{-}^{d}(t), q(t-)\right), & \text { if } q_{0}(t), \\
\mathfrak{S}\left(\dot{e}(t), \varphi_{+}^{d}(t), \max \left\{\dot{\varphi}_{-}(t), 0\right\}, q(t-)\right), & \text { else, }\end{cases}
\end{aligned}
$$

where $\mathfrak{S}$ is the switching predicate as given in (6). The switching logic (applied to the control law (3)) is illustrated as a simplified state diagram in Figure 5.

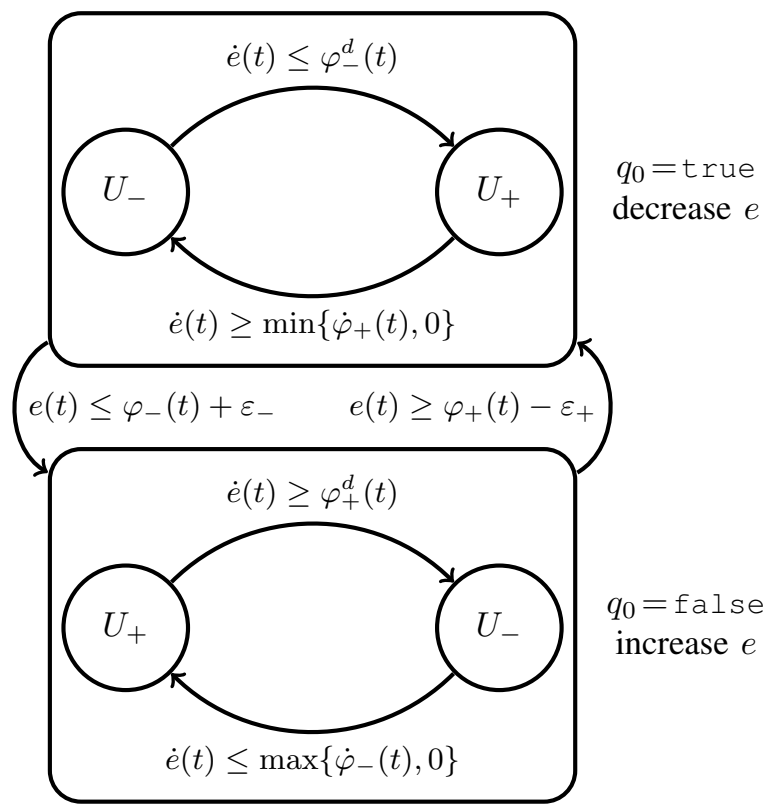

Fig. 5. The switching logic for the relative degree two case.

The reasoning behind the switching logic (14) is as follows (see also the schematic illustration in Figure 6): Whenever the error gets close to the upper funnel boundary, i.e. $e(t) \geq$ $\varphi_{+}(t)-\varepsilon_{+}$, we would like to decrease $e(t)$, a task which is encoded by $q_{0}(t)=$ true. To do this we have to decrease $\dot{e}$ which under certain feasibility assumptions is possible by applying $u(t)=U_{-}$. It will take some time until $\dot{e}$ is small enough, which is the case when $\dot{e}(t) \leq \dot{\varphi}_{+}(t)$ because then the distance to the upper funnel boundary starts increasing. At this 


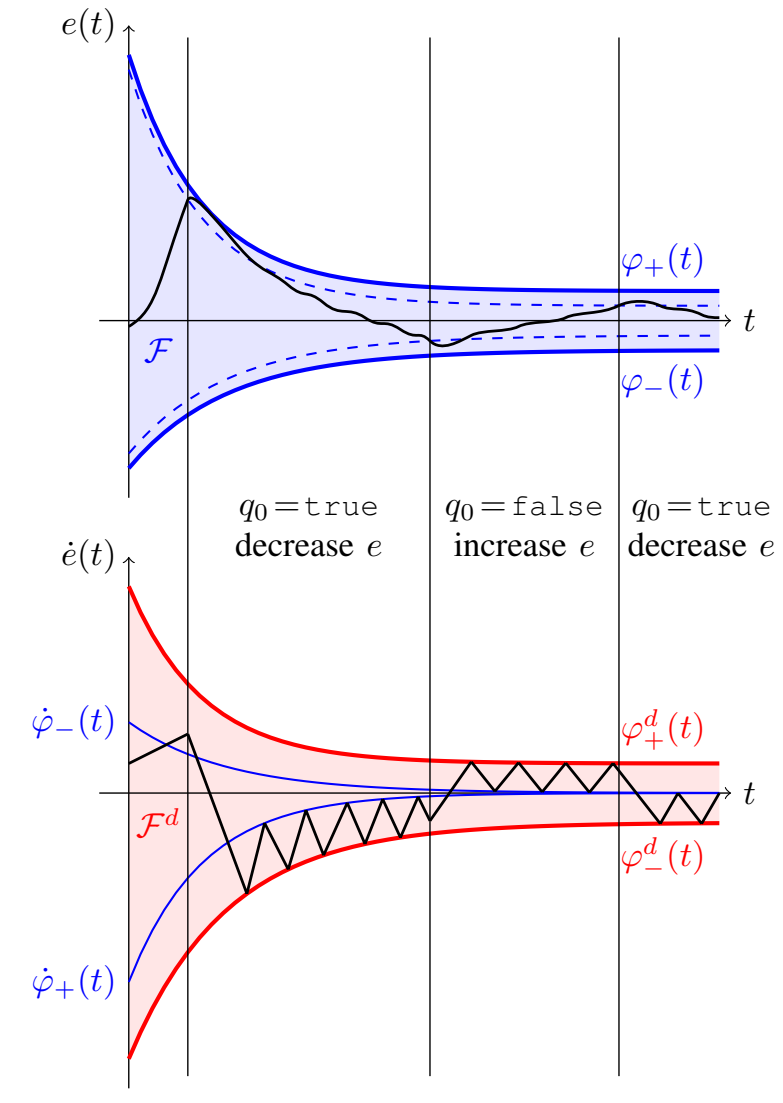

Fig. 6. A schematic illustration how the error and its derivative evolve under the switching logic (14), the vertical lines indicate the switching of the predicate $q_{0}$ which indicates wether we would like to decrease or increase the error.

point we could keep $u(t)=U_{-}$until the error gets close to the lower funnel boundary. However, this would unnecessarily decrease $\dot{e}(t)$ further, which implies that when we want to increase the error later (when we got close the lower funnel boundary) it will take longer until $\dot{e}(t)$ is big enough so that the distance of $e(t)$ to the lower funnel boundary is increasing. That is why we stop decreasing $\dot{e}$ by setting $u(t)=U_{+}$when the lower derivative funnel boundary is hit, i.e. when $\dot{e}(t) \leq$ $\varphi_{-}^{d}(t)$. If we still want to decrease the error, we have to stop increasing the derivative of the error when $\dot{e}(t) \geq \dot{\varphi}_{+}(t)$ or $\dot{e}(t) \geq 0$.

A similar result as Lemma 2.2 holds also for the relative degree two case.

Lemma 3.2 (Well-defined causal switching logic): For every continuously differentiable error function $e:[0, T) \rightarrow \mathbb{R}$, $0<T \leq \infty$ there exists a unique causal right-continuous switching signal $q:[0, T) \rightarrow\{$ true, false $\}$ fulfilling (14). If additionally $\dot{e}$ is bounded and absolutely continuous with right-continuous bounded derivative $\ddot{e}$ and

$$
0<\lambda \wedge 0<\tau_{+}(\|\ddot{e}\|) \wedge 0<\tau_{-}(\|\ddot{e}\|)
$$

where $\lambda:=\inf _{t \geq 0} \varphi_{+}(t)-\varepsilon_{+}-\sup _{t \geq 0} \varphi_{-}(t)-\varepsilon_{-}, \tau_{+}(\delta):=$ $\inf _{t \geq 0} \inf \left\{|\tau| \mid \varphi_{+}^{d}(t)-\max \left\{\dot{\varphi}_{-}(t+\tau), 0\right\} \leq \tau \delta\right\} \quad$ and $\tau_{-}(\bar{\delta}):=\inf _{t \geq 0} \inf \left\{|\tau| \mid \min \left\{\dot{\varphi}_{+}(t), 0\right\}-\varphi_{-}^{\bar{d}}(t+\tau) \leq \tau \delta\right\}$ then the switching signal has an average dwell time [15]

$$
\tau_{a} \geq \frac{1}{\frac{\|\dot{e}\|}{\lambda}+\min \left\{\tau_{+}(\|\ddot{e}\|), \tau_{-}(\|\ddot{e}\|)\right\}}
$$

with chattering bound two, i.e. the number of switches $N(t, T)$ in every time interval $[t, T)$ is bounded by $2+\frac{T-t}{\tau_{a}}$.

Corollary 3.3 (Closed loop well posed): Consider system (1) with relative degree two in closed loop with the bang-bang controller given by (14) and (3) where $e:=y-y_{\text {ref }}$ for some continuously differentiable reference signal $y_{\text {ref }}: \mathbb{R}_{\geq 0} \rightarrow \mathbb{R}$. Then for every initial value $x^{0} \in \mathbb{R}^{n}$ there exists a unique maximal solution $\left(x, q_{0}, q\right):[0, \omega) \rightarrow \mathbb{R}^{n} \times\{$ true, false $\} \times$ \{true, false $\}, 0<\omega \leq \infty$.

As in the relative degree one case, Corollary 3.3 does not assume or claim that the error evolves within the funnel. For this some additional feasibility assumptions are needed.

Theorem 3.4 (Relative degree two case main result):

Assume that (1) has relative degree two, i.e. (1) is equivalent to (10). Consider a funnel $\mathcal{F}$ as given by (2) whose differentiable boundary functions $\varphi_{ \pm}: \mathbb{R}_{\geq 0} \rightarrow \mathbb{R}$ have absolutely continuous derivatives with right-continuous second derivatives and fulfill (13) for some $\varepsilon_{ \pm}>0$. Choose a derivative funnel $\mathcal{F}^{d}$ as in (11) whose funnel boundaries $\varphi_{ \pm}^{d}: \mathbb{R}_{\geq 0} \rightarrow \mathbb{R}$ are absolutely continuous with right-continuous derivative and fulfill assumption (12). Let $y_{\text {ref }}: \mathbb{R}_{\geq 0} \rightarrow \mathbb{R}$ be a differentiable reference signal whose derivative is absolutely continuous with right-continuous second derivative. Assume that the initial conditions for (10) fulfill

$$
\begin{aligned}
& y^{0}-y_{\mathrm{ref}}(0) \in\left[\varphi_{-}(0)+\varepsilon_{-}, \varphi_{+}(0)-\varepsilon_{+}\right], \\
& \dot{y}^{0}-\dot{y}_{\mathrm{ref}}(0) \in\left[\varphi_{-}^{d}(0), \varphi_{+}^{d}(0)\right], \quad z^{0} \in Z_{0} \subseteq \mathbb{R}^{n-2}
\end{aligned}
$$

and assume that for every differentiable $y:[0, \infty) \rightarrow \mathbb{R}$ with $\varphi_{-}(t) \leq y(t)-y_{\text {ref }}(t) \leq \varphi_{+}(t), \varphi_{-}^{d}(t) \leq \dot{y}(t)-\dot{y}_{\text {ref }}(t) \leq$ $\varphi_{+}^{d}(t)$ and for every initial value $z^{0} \in Z_{0}$ there exists a unique (global) solution $z: \mathbb{R}_{\geq 0} \rightarrow \mathbb{R}^{n-2}$ of the zero dynamics (10b); for $t>0$ let

$$
Z_{t}:=\left\{\begin{array}{l|l}
z(t) & \begin{array}{l}
z:[0, t] \rightarrow \mathbb{R}^{n-1} \text { solves }(10 \mathrm{~b}) \text { for some } \\
z^{0} \in Z_{0} \text { and for some } y:[0, t] \rightarrow \mathbb{R} \text { with } \\
\varphi_{-}(\tau) \leq y(\tau)-y_{\mathrm{ref}}(\tau) \leq \varphi_{+}(\tau) \text { and } \\
\varphi_{-}^{d}(\tau) \leq \dot{y}(\tau)-\dot{y}_{\mathrm{ref}}(\tau) \leq \varphi_{+}^{d}(\tau) \forall \tau \in[0, t]
\end{array}
\end{array}\right\} .
$$

If there exist $\delta_{ \pm}>0$ with

$$
\begin{gathered}
\delta_{+}>\max \left\{\dot{\varphi}_{-}^{d}(t), \ddot{\varphi}_{-}(t), 0\right\} \quad \text { and } \\
-\delta_{-}<\min \left\{\dot{\varphi}_{+}^{d}(t), \ddot{\varphi}_{+}(t), 0\right\} \quad \forall t \geq 0
\end{gathered}
$$

such that the first set of feasibility conditions

$$
\begin{aligned}
& U_{-}<\frac{-\delta_{-}+\ddot{y}_{\mathrm{ref}}(t)+f\left(y_{t}, \dot{y}_{t}, z_{t}\right)}{g\left(y_{t}, \dot{y}_{t}, z_{t}\right)}, \\
& U_{+}>\frac{\delta_{+}+\ddot{y}_{\mathrm{ref}}(t)+f\left(y_{t}, \dot{y}_{t}, z_{t}\right)}{g\left(y_{t}, \dot{y}_{t}, z_{t}\right)}
\end{aligned}
$$

hold for all $t \geq 0$ and all $\left(y_{t}, \dot{y}_{t}, z_{t}\right) \in\left[y_{\text {ref }}(t)+\varphi_{-}(t), y_{\text {ref }}(t)+\right.$ $\left.\varphi_{+}(t)\right] \times\left[\dot{y}_{\mathrm{ref}}(t)+\varphi_{-}^{d}(t), \dot{y}_{\mathrm{ref}}(t)+\varphi_{+}^{d}(t)\right] \times Z_{t}$, and if the second set of feasibility conditions

$$
\varepsilon_{+} \geq \frac{\left(\left\|\varphi_{-}^{d}\right\|+\left\|\min \left\{\dot{\varphi}_{+}, 0\right\}\right\|\right)^{2}}{2 \delta_{-}}, \quad \varepsilon_{-} \geq \frac{\left(\left\|\varphi_{+}^{d}\right\|+\left\|\max \left\{\dot{\varphi}_{-}, 0\right\}\right\|\right)^{2}}{2 \delta_{+}}
$$

hold then the bang-bang controller (3) governed by the switching logic (14) applied to (1) or, equivalently, (10) achieves the control objectives, i.e. the closed loop has the following 
properties:

1) There exists a (global) unique solution $\left(x, q_{0}, q\right)$ : $\mathbb{R}_{\geq 0} \rightarrow \mathbb{R}^{n} \times\{$ true, false $\times\{$ true, false $\}$.

2) The error $e:=y-y_{\text {ref }}$ evolves within the funnel $\mathcal{F}$ and the derivative of the error $\dot{e}$ evolves within the derivative funnel $\mathcal{F}^{d}$, i.e. $(t, e(t)) \in \mathcal{F}$ and $(t, \dot{e}(t)) \in \mathcal{F}^{d}$ for all $t \geq 0$.

3) If $f$ and $g$ are uniformly bounded on $\bigcup_{t>0}\left[y_{\text {ref }}(t)-\right.$ $\left.\varphi_{i}(t), y_{\mathrm{ref}}(t)+\varphi_{+}\right] \times\left[\dot{y}_{\mathrm{ref}}+\varphi_{-}^{d}, \dot{y}_{\mathrm{ref}}+\varphi_{+}^{d}\right] \times Z_{t}, \ddot{y}_{\mathrm{ref}}$ is bounded, $\varphi_{ \pm}^{d}$ are bounded and (15) holds for all $\delta>0$ then the switching signal $q$ has a positive average dwell time $\tau_{a}>0$.

Remarks 3.5: 1) The two main results, Theorem 2.4 and Theorem 3.4, do not depend on the initialization $q(0-)$ and $q_{0}(0-)$ for the switching logic. However, the choice in (5) and (14) intuitively improves performance, because the control action is in the "right" direction just from the start and not only after the first boundary is hit.

2) The second feasibility assumption (17) might be in contradiction with the assumption (13). However, increasing/decreasing $U_{ \pm}$(without changing anything else) allows for bigger $\delta_{ \pm}$so that (17) yields arbitrarily small lower bounds for $\varepsilon_{ \pm}$and (13) is not in contradiction with (17) anymore.

3) As for the relative degree one case it is possible to simplify the feasibility assumption (16) by considering upper bounds for the funnel boundaries (and their derivatives), the zero dynamics, and the reference signal (and its derivatives). In particular, for minimum phase linear systems with relative degree two it follows then that (16) holds whenever $U_{-}<0$ and $U_{+}>0$ are large enough.

4) The feasibility assumptions could possibly be made less conservative by introducing time-varying safety distances $\varepsilon_{ \pm}(t)$. Typically the funnels are large with large derivatives at the beginning, hence require larger safety distances by (17), and on the other hand tighter funnels with small derivatives later in time restrict the size of the safety distance by (13) although, at least locally, (17) does not require big safety distances anymore.

5) The first feasibility assumption (16) looks very similar to the feasibility assumption in Theorem 2.4 applied to $\dot{e}$ and $\mathcal{F}^{d}$. The two main differences are that, firstly, $\dot{\varphi}_{ \pm}^{d}$ are replaced by uniform lower/upper bounds $\delta_{\mp}$ and, secondly, (16) has to hold on the whole funnel region and not only on the boundary. The reason for both is that we need a certain minimum decrease/increase of $\dot{e}$ in the whole funnel (and not only on the boundary) to ensure that we can quantify the overshoot of $e$ (in fact, condition (17) is this quantification).

6) The switching logic for the relative degree two case is hierarchically composed, where the outer switching logic is identical (apart from the safety distance) to the switching logic of the relative degree one case. The authors were already able to define a switching logic for the relative degree three case based on a hierarchical composition similar to the one presented here, but due to space limitations this result is not included here. In fact, it seems much more interesting to come up with a general solution for an arbitrary relative degree; this is ongoing research.

\section{CONCLUSIONS}

A universal controller was proposed which only uses two input values and is governed by a simple switching logic. This switching logic depends on the relative degree of the system, otherwise no knowledge of the system is necessary to design the controller. Feasibility assumptions are given which ensure that approximate reference signal tracking with strict timevarying error bounds is achieved. We assumed that the gain function $g$ in the relative degree normal forms is positive; however, it should be possible to extend the results to an unknown (but definite) sign of the gain function by slightly changing the switching logic to first detect the sign of the gain function.

The nature of the controller seems to make it more "robust" than the continuous funnel controller because, in contrast to the latter, the bang-bang funnel controller is still well defined when the error leaves the funnel, for example when a time delay is present. A precise robustness result is a future research topic.

The switching logic for the relative degree two case already hints to switching logics for higher relative degrees; this is a topic of ongoing research.

\section{ACKNOWLEDGEMENTS}

We thank Achim Ilchmann for giving valuables comments on the manuscript of this paper.

\section{REFERENCES}

[1] A. Ilchmann, E. P. Ryan, and C. J. Sangwin, "Tracking with prescribed transient behaviour," ESAIM: Control, Optimisation and Calculus of Variations, vol. 7, pp. 471-493, 2002.

[2] A. Ilchmann and E. P. Ryan, "High-gain control without identification: a survey," GAMM Mitt., vol. 31, no. 1, pp. 115-125, 2008.

[3] A. Ilchmann, E. P. Ryan, and S. Trenn, "Tracking control: Performance funnels and prescribed transient behaviour," Syst. Control Lett., vol. 54, no. 7, pp. 655-670, 2005.

[4] A. Ilchmann, E. P. Ryan, and P. Townsend, "Tracking control with prescribed transient behaviour for systems of known relative degree," Syst. Control Lett., vol. 55, no. 5, pp. 396-406, 2006.

[5] -, "Tracking with prescribed transient behavior for nonlinear systems of known relative degree," SIAM J. Control Optim., vol. 46, no. 1, pp. 210-230, 2007. [Online]. Available: http://link.aip.org/link/?SJC/46/210/1

[6] D. E. Miller and E. J. Davison, "An adaptive controller which provides an arbitrarily good transient and steady-state response," IEEE Trans. Autom. Control, vol. 36, no. 1, pp. 68-81, 1991.

[7] A. Ilchmann and S. Trenn, "Input constrained funnel control with applications to chemical reactor models," Syst. Control Lett., vol. 53, no. 5, pp. 361-375, 2004.

[8] N. Hopfe, A. Ilchmann, and E. P. Ryan, "Funnel control with saturation: linear MIMO systems," IEEE Trans. Autom. Control, vol. 55, no. 2, pp. 532-538, 2010.

[9] _ "Funnel control with saturation: nonlinear SISO systems," 2010, conditionally accepted for IEEE Trans. Autom. Control.

[10] C. Hackl, N. Hopfe, A. Ilchmann, M. Mueller, and S. Trenn, "Funnel control for systems with relative degree two," 2010, submitted for publication, preprint available from the webpages of the authors.

[11] D. Liberzon, Switching in Systems and Control, ser. Systems and Control: Foundations and Applications. Boston: Birkhäuser, 2003.

[12] J. Lygeros, C. Tomlin, and S. Sastry, "Controllers for reachability specifications for hybrid systems," Automatica, vol. 35, no. 3, pp. 349370, March 1999.

[13] D. Liberzon and S. Trenn, "The bang-bang funnel controller (long version)," 2010, preprint available from the websites of the authors.

[14] A. Isidori, Nonlinear Control Systems, 3rd ed., ser. Communications and Control Engineering Series. Berlin: Springer-Verlag, 1995.

[15] J. P. Hespanha and A. S. Morse, "Stability of switched systems with average dwell-time," in Proc. 38th IEEE Conf. Decis. Control, 1999, pp. 2655-2660. 